\title{
How Astrocytes Feed Hungry Neurons
}

\section{Luc Pellerin}

\author{
Département de Physiologie, Université de Lausanne, Switzerland
}

\begin{abstract}
For years glucose was thought to constitute the sole energy substrate for neurons; it was believed to be directly provided to neurons via the extracellar space by the cerebral circulation. It was recently proposed that in addition to glucose, neurons might rely on lactate to sustain their activity. Therefore, it was demonstrated that lactate is a preferred oxidative substrate for neurons not only in vitro but also in vivo. Moreover, the presence of specific monocarboxylate transporters on neurons as well as on astrocytes is consistent with the hypothesis of a transfer of lactate from astrocytes to neurons. Evidence has been provided for a mechanism whereby astrocytes respond to glutamatergic activity by enhancing their glycolytic activity, resulting in increased lactate release. This is accomplished via the uptake of glutamate by glial glutamate transporters, leading to activation of the $\mathrm{Na}^{+} / \mathrm{K}^{+}$ATPase and a stimulation of astrocytic glycolysis. Several recent observations obtained both in vitro and in vivo with different approaches have reinforced this view of brain energetics. Such an understanding might be critically important, not only because it forms the basis of some classical functional brain imaging techniques but also because several neurodegenerative diseases exhibit diverse alterations in energy metabolism.
\end{abstract}

Index Entries: Energy metabolism; glucose; lactate; functional brain imaging; neurodegenerative diseases; neurometabolic coupling.

\section{Introduction}

The brain represents only 2 to $3 \%$ of the body mass, but it receives $15 \%$ of the blood supply and consumes $25 \%$ of all glucose at rest as well as $20 \%$ of all oxygen. These observa-

Received 10/18/04; Accepted 12/17/04.

Author to whom all correspondence and reprint requests should be addressed. E-mail: Luc.Pellerin@ iphysiol.unil.ch. tions emphasize the importance of brain energy requirements to sustain cerebral activity. Therefore, it is of crucial importance to understand how this energy is generated and which processes require it as well as to decipher the mechanisms that regulate these processes. In the past $10 \mathrm{yr}$, important changes have occurred regarding our understanding of neuroenergetics (or the different aspects related to brain energy metabolism). This article reviews several of the key aspects that have emerged during that period. 


\section{Classical Neuroenergetics: The Central Dogma}

Years of investigation in the field of brain energy metabolism have led to a large consensus regarding some rules forming what was believed to be the unquestionable basis of neuroenergetics, or how energy is produced and used to support brain function. Such rules constitute the so-called "central dogma" of neuroenergetics and can be stated as follows:

- Energy costs in the central nervous system (CNS) are dominated by neuronal activity (approx $80-95 \%)$, whereas glial cells account for only a small fraction (approx 5-20\%).

- Glucose is the sole energy substrate used to support function of the adult brain.

- Complete oxidation of glucose provides all the energy necessary to sustain brain activity.

- Glucose utilization is tightly coupled to synaptic activity.

Each of these points deserves further elaboration. First, recent calculations and nuclear magnetic resonance (NMR) measurements have estimated the relative contribution of neurons and glia to overall energy expenditure of the brain as well as the specific cost associated with different processes involved in brain function $(1,2)$. Based on this evaluation, only about 5 to $20 \%$ of all energy expenditure would result from glial cells, whereas the rest would be directly related to neuronal activity. This ratio between neuronal and glial energy costs is in agreement with to the reported distribution of mitochondria between the two cell types (98 vs $2 \%$ in neurons and astrocytes, respectively; ref. 3). If it is considered that glucose oxidation is the major source of energy for the CNS, then it is no surprise that the distribution of mitochondria corresponds to cellular energy needs.

A classical statement found in biochemical textbooks says that the brain relies solely on blood-borne glucose to provide all the energy necessary to support brain function. Although there appears to be no doubt that glucose represents an essential and major energy substrate for the brain, there are a few situations for which other substrates can provide a significant amount of energy to fulfill part of the brain energy requirements. This is especially the case during brain development. In the first few hours following delivery, lactate was found to be an important energy substrate for the brain, whereas ketone bodies sustain brain function during the entire preweaning period (4). Despite these so-called "exceptions," glucose must still be considered the major, if not the exclusive, energy substrate for the adult brain and, by extension, for satisfying neuronal energy needs.

Parallel measurements of oxygen consumption and glucose utilization have shown a ratio of approx 5.5, a value close to the predicted ratio of 6 if glucose was entirely oxidized. Moreover, the cerebral respiratory quotient that reflects the ratio between $\mathrm{CO}_{2}$ production over $\mathrm{O}_{2}$ consumption was estimated to be 1.0. These global measurements performed on the resting brain indicate that carbohydrates, and most likely glucose, are the major energy substrate for the brain and that oxidative phosphorylation represents the major pathway by which adenosine triphosphate (ATP) is generated in the brain.

Such observations give relatively little importance to other substrates and pathways as sources of energy. However, there exists much debate surrounding a transient change in this ratio after specific paradigms of activation. This situation, called uncoupling, was first described by Raichle and collaborators (5). These authors described that an increase in glucose utilization (and blood flow) in the activated area of the brain (in this case, the visual cortex during intense visual stimulation) is not accompanied by a commensurate (and proportional) increase in oxygen consumption. Although such a phenomenon can be clearly observed in specific circumstances, it remains to be determined whether this is the exception or the rule.

Concerning the last point of the dogma, it was assumed that the mechanism subserving a tight link between glucose utilization and 


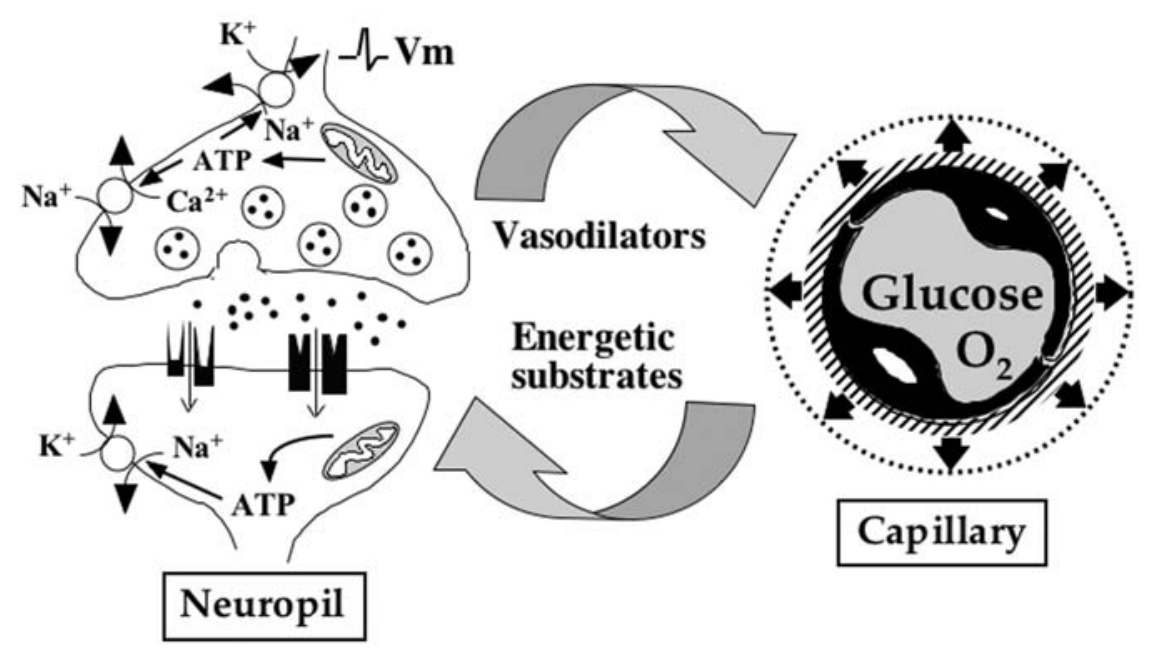

Fig. 1. Classical view of how blood flow, glucose utilization, and oxygen consumption are linked to synaptic activity. Imbalances in ion homeostasis caused by the propagation of action potentials, the release of neurotransmitters, and postsynaptic potentials lead to ATP consumption and create important energy needs in neurons. To cope with this increased energy demand, vasodilators are produced, the identity of which are still debated. Because of the formation of vasodilators, an increased blood flow ensues in the activated area, bringing more glucose and oxygen. Both glucose and oxygen cross the blood-brain barrier (via facilitating transporters and by simple diffusion, respectively) and reach the extracellular space to be directly taken up by active neurons. Glucose oxidation in neurons provides the necessary ATP to re-establish ion gradients and maintain excitability.

synaptic activity was relatively well-understood and could be summarized as follows (Fig. 1). Increased neuronal activity in any brain region is accompanied by the formation and release of vasoactive substances, leading to a local increase in blood flow. Although the identity of these substances, their origin, and their modes of action are varied and their respective roles are much debated, the end result is an enhanced blood flow, allowing more glucose and oxygen to be carried to the active area. After crossing the blood-brain barrier (glucose via specific tranporters such as glucose transporter-1 [55-kDa GLUT1] and oxygen via simple diffusion), these two substances penetrate the brain parenchyma and become directly available to active neurons to be used and fulfill neuronal energy needs. In this oversimplified description of the purported neurometabolic coupling mechanism, the putative contribution of glial cells has never been considered.

\section{Astrocytes Exhibit Features That Make Them Ideal for a Nurturing Role Toward Neurons}

Some important cellular elements have not been considered in the classical description of the coupling mechanism between glucose utilization and neuronal activity (illustrated in Fig. 1); this is the case of astrocytes. Indeed, these cells occupy a strategic position, interposed between blood vessels, which represent the source of glucose, and neurons, which are the main energy consumers within the brain parenchyma. Moreover, astrocytes exhibit a certain number of cytoarchitectural characteristics that suggest a putative role in the regulation of brain energy metabolism (Fig. 2). They project toward blood vessel processes that terminate into structures called endfeet, which almost entirely cover the blood vessel walls. 


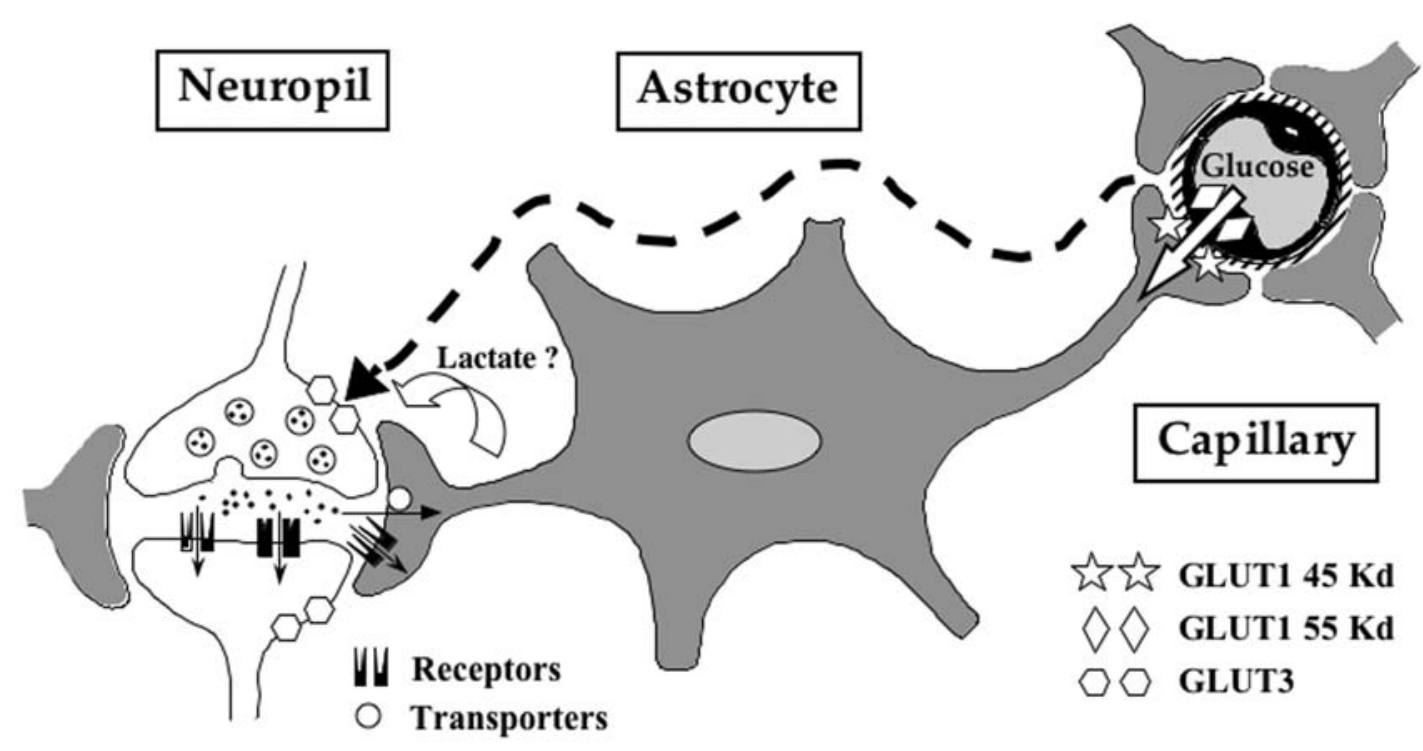

Fig. 2. Proposed role of astrocytes in coupling synaptic activity to glucose utilization. Astrocytic endfeet cover the surface of capillaries, and these processes express the glucose transporter GLUT1. In parallel, fine astrocytic lamellae (which can express receptors and transporters for almost any neurotransmitter) ensheath synapses. These cytoarchitectual characteristics suggest that astrocytes are ideally located and equipped to couple synaptic activity to glucose utilization. To compensate for the use of glucose by astrocytes, these cells release a metabolic intermediate to be used by neurons as an additional energy substrate. The most likely candidate for this role appears to be lactate, which can be produced in large quantities by astrocytes and can be significantly consumed by neurons.

On the membrane of endfeet facing blood vessels, astrocytes express a specific form of glucose transporters: 45-kDa GLUT1 $(6,7)$.

This anatomical arrangement, together with the expression of glucose transporters, suggests that astrocytes might represent a privileged uptake site for glucose as it leaves the circulation to enter the brain parenchyma, as suggested by Golgi more than 100 yr ago (8). On the other hand, astrocytes also extend processes that contact neurons and, particularly, enclose synapses. These processes express receptors and/or transporters for one or the other neurotransmitter (9-12). Such characteristics endow astrocytes with the capacity to detect synaptic activity and, possibly, transduce it into a specific message (13). Recent evidence indicates that astrocytes can respond to synaptic activity by releasing themselves, glutamate, or ATP that in turn would modulate neuronal activity (14).
Accumulating evidence also suggests that increased synaptic activity could lead to a concomitant metabolic response in astrocytes (15).

A series of experiments performed in vitro on primary cultures of astrocytes characterized a putative mechanism that might at least partially account for the observed coupling between synaptic activity and glucose utilization in vivo. Glutamate (the major excitatory neurotransmitter in the $\mathrm{CNS}$ ) was shown to enhance glucose utilization in cultured astrocytes, as measured by the uptake of the glucose analog 2-deoxyglucose (16). This effect did not result from the activation of glutamate receptors, but rather from the uptake of glutamate via specific $\mathrm{Na}^{+}$-dependent glutamate transporters located on astrocytes. Consequently, an increase in the intracellular $\mathrm{Na}^{+}$concentration occurred (17), leading to an activation of the $\mathrm{Na}^{+} / \mathrm{K}^{+}$ATPase (18). Moreover, it was concluded that a specific 
subunit of the $\mathrm{Na}^{+} / \mathrm{K}^{+}$ATPase (akin to the $\alpha_{2}-$ subunit) was mobilized in these circumstances (18). Interestingly, the $\alpha_{2}$-subunit of the $\mathrm{Na}^{+} / \mathrm{K}^{+}$ ATPase was found to be specifically expressed by astrocytes in vivo and colocalized with both glial glutamate aspartate transporter (GLAST) and glutamate transporter-1 (GLT1) on fine astrocytic processes surrounding glutamatergic synapses (19).

Based on indirectly measured alterations in ATP levels on exposure to glutamate in cultured astrocytes (20), it has been suggested that ATP consumption by the $\mathrm{Na}^{+} / \mathrm{K}^{+}$ATPase via an alteration of the ATP/adenosine diphosphate ratio disinhibits glycolysis and causes an increase in glucose utilization. In parallel with this effect on glucose utilization, it was recently demonstrated that glutamate causes a reversible enhancement in glucose transport that takes place within a few seconds after the beginning of glutamate exposure (21). Therefore, there appears to be a concerted mechanism to increase both glucose uptake and utilization in astrocytes after glutamate exposure.

The metabolic fate of glucose taken up by astrocytes upon exposure to glutamate has also been investigated. A large part of the additional glucose taken up is converted to lactate and released by astrocytes $(16,22)$. Although oxidation of pyruvate via the Krebs cycle is a more efficient pathway regarding ATP production, the reason that astrocytes favor the formation of lactate is not completely understood. To sustain a high glycolytic flux (as observed in glutamate-stimulated astrocytes), the cytosolic pool of nicotinamide adenine dinucleotide $\left(\mathrm{NAD}^{+}\right)$ essential at the step catalyzed by the glyceraldehyde-3-phosphate dehydrogenase must be rapidly regenerated. It has been proposed that the cytosolic pool of $\mathrm{NAD}^{+}$would be more rapidly and efficiently maintained by converting pyruvate to lactate (which requires one $\mathrm{NADH}$ and gives one $\mathrm{NAD}^{+}$) via the reaction catalyzed by the lactate dehydrogenase (LDH) present in the cytosol. An additional argument that has emerged recently is linked to the observation that astrocytes apparently lack a mitochondrial aspartate-glutamate carrier, which is an essential component of the malate-aspartate shuttle (23). As a consequence, astrocytes would have a reduced capacity to shuttle NADH from the cytosolic to the intramitochondrial compartment and to regenerate the cytosolic $\mathrm{NAD}^{+}$pool via oxidative metabolism. Therefore, this would result in their preference for pyruvate conversion to lactate with regeneration of $\mathrm{NAD}^{+}$within the cytosol.

Some investigators have suggested that the glycolytic response observed in astrocytes after exposure to glutamate could be an artifact arising from culture conditions $(24,25)$. They contended that high glucose $(25 \mathrm{mM})$ concentration in culture media could favor the emergence of a glycolytic phenotype in cultured cells and prevents the oxidative use of glutamate to support its own uptake. A clear demonstration that enhanced aerobic glycolysis following glutamate exposure is a constitutive property of astrocytes independent of glucose concentrations was provided recently (26). Therefore, cultured astrocytes prepared from mouse neural stem cells and cultured from the beginning in the presence of either 33 or $5 \mathrm{mM}$ displayed the same glycolytic response to glutamate. When cultured under the same conditions, undifferentiated stem cells also had a much less glycolytic phenotype compared to astrocytes, as revealed by their very low basal lactate production. Moreover, the capacity of astrocytes to enhance aerobic glycolysis after glutamate exposure appeared with their differentiation. The acquisition of such a metabolic response appears linked to a particular cell phenotype and is not caused by cell culture conditions. This conclusion is strengthened by ex vivo and in vivo observations demonstrating the existence of a glycolytic response in astrocytes after brain activation, as described in the following section.

\section{Ex Vivo and In Vivo Evidence for the Involvement of Astrocytes in Neurometabolic Coupling}

Critical evidence for a role of astrocytes in neurometabolic coupling based on ex vivo 
and in vivo experiments has been provided recently. Using a specific approach with two photon confocal microscopy to monitor NADH fluorescence, Kasischke and coworkers (27) demonstrated that in hippocampal slices, astrocytes in the CA1 area responded to afferent stimulation via Schaffer collaterals with an enhanced cytosolic NADH signal corresponding to an activation of glycolysis. This response was not prevented by glutamate receptor antagonists, leaving the possibility that it could have been mediated via glutamate uptake. In vivo, it was shown that injection of antisense oligonucleotides against the glial glutamate transporter GLAST in the adult rat barrel cortex partly prevents the enhanced deoxyglucose accumulation observed in the corresponding cortical column following whisker stimulation (28). Moreover, using the same whisker-to-barrel model system, an approx $60 \%$ reduction in deoxyglucose accumulation in the appropriate barrel was observed in 10-d-old knockout mice for either GLAST or GLT1; this effect persisted in adult GLT1 -/mice $(29,30)$. These data suggest that a significant proportion of the enhanced glucose utilization after activation of a specific brain area occurs in astrocytes.

This observation appears to contradict the central dogma. Indeed, if glucose is the only significant energy substrate for the brain (being entirely oxidized to provide all the ATP necessary to sustain brain function) and if only 5 to $20 \%$ of the energy requirements come from astrocytes, then it should be expected that no more than $20 \%$ of the enhanced glucose utilization depend on astrocytes. Such a major departure from the predicted distribution in glucose utilization between neurons and glial cells has been reported in two other cases.

Nehlig and coworkers (31) recently established a new method combining immunocytochemistry with high-resolution microautoradiography that allowed them to quantitate the distribution of accumulated radioactive deoxyglucose between astrocytes and neurons in the hippocampus of resting animals. Their results indicate that about half of all glucose utilization under these conditions occurs in astrocytes. During activation, this ratio might even increase in favor of astrocytes, as suggested by in vitro experiments measuring glucose uptake after glutamate exposure in both cell types $(21,32)$ as well as in the aforementioned experiments in glial glutamate transporter knockout mice $(29,30)$.

Considering that the same metabolic requirements are likely shared between the CNS and peripheral nervous system (33), ex vivo experiments performed on a stimulated vagus nerve preparation have provided an interesting insight regarding this issue. Evaluation of glucose uptake between axons and Schwann cells upon stimulation of the axon led to the unexpected conclusion that $78 \%$ of glucose utilization occurs in Schwann cells, the astrocyte equivalent for the peripheral nervous system (34). Altogether, results of these studies are inconsistent with the previously established rules.

One way to reconcile this paradox is to consider that a fraction of glucose is only partially metabolized in glial cells and that an intermediate is released, which is to be used by neighboring neurons. Evidence points at lactate as the most likely candidate (34-36). Therefore, considering the ATP yield of glycolysis for one glucose in astrocytes (2 ATP) and of oxidative metabolism from two lactates in neurons (approx 30 ATP), a transfer of lactate between astrocytes and neurons still allows fulfillment of the predicted energy needs of the two cell types and explains the importance of their respective glucose utilization as measured experimentally. Recent studies using NMR spectroscopy have provided a clear demonstration that a net lactate transfer occurs between astrocytes and neurons within the brain and augments with increasing levels of activity $(37,38)$.

\section{Lactate Represents a Preferential Oxidative Energy Substrate for Neurons}

In the past $50 \mathrm{yr}$, a wide array of studies using different approaches have led to a similar conclusion: lactate is efficiently used oxidatively by the nervous tissue (for review, see ref. 
39). Therefore, experiments performed on brain slices (40-42), cultured telencephalic neurons (43-45), aggregated neuronal cultures (46), sympathetic ganglia (47-50), and synaptic terminals (51-53) have consistently reported that lactate can be oxidized to $\mathrm{CO}_{2}$ by these preparations-usually more efficiently than glucose. A direct demonstration that neurons preferentially use lactate over glucose as an oxidative substrate was recently provided with two distinct approaches. By measuring ${ }^{14} \mathrm{CO}_{2}$ produced by cultured neurons from ${ }^{14} \mathrm{C}$ labeled substrates, Sokoloff and colleagues (54) demonstrated that these cells, in contrast to astrocytes, have a kinetic preference for oxidation of extracellular lactate over pyruvate produced intracellularly from glucose. A similar demonstration was provided using NMR spectroscopy. When both substrates were present at equimolar concentration $(5.5 \mathrm{mM})$, it was calculated that nearly $90 \%$ of neuronal oxidative metabolism was supported by lactate (55). Evidence that lactate represents a significant energy substrate for the adult brain have also been provided. Thus, NMR spectroscopy showed that intravenously injected lactate was readily metabolized by the brain in a compartment lacking pyruvate carboxylase activity $(56,57)$. Because pyruvate carboxylase activity is found only in astrocytes, it was suggested that lactate utilization occurs predominantly in neurons. Additionally, it was concluded from the labeling pattern of compounds such as glutamine, glutamate, and $\gamma$-aminobutyric acid (GABA) that lactate must be used in large part by glutamatergic neurons (58). An in vivo confirmation of the preferred use of lactate over glucose was recently obtained in human subjects. Amiel and coworkers (59) showed that raising plasma lactate levels to values reached during a moderate exercise substantially reduced brain glucose utilization, as revealed by the use of labeled fluorodeoxyglucose combined with positron emission tomography.

Although several studies have indicated that lactate could be an important energy substrate for neurons under resting conditions, few have examined changes in substrate utilization on stimulation. Two interesting observations were made recently. First, using fluorescent analogs of deoxyglucose and confocal microscopy, it was demonstrated that glutamate exposure induces opposite effects in astrocytes and neurons within the same culture preparation: although glutamate increased glucose transport in astrocytes, it reduced it in neurons (32). Indeed, the ratio of glucose transport between astrocytes and neurons varied from 1.27:1 at rest to 15.8:1 after addition of glutamate. This change in neurons occurs within a few seconds, depends on the activation of AMPA receptors, and is fully reversible upon glutamate removal.

Therefore, it appears that glutamate favors glucose uptake in astrocytes and restricts its entry into neurons. These conditions highly contribute to promotion of lactate production by astrocytes and its oxidative use by neurons. The latter point is supported by the observation that addition of lactate to the medium concomitantly with glutamate further reduces glucose uptake in neurons (32).

Another set of experiments performed in hippocampal slices provided an interesting insight regarding this issue. Kasischke and colleagues (27) observed a rapid activation of oxidative metabolism in dendrites of CA1 neurons upon Schaffer collateral activation, which was followed by enhanced glycolysis in neighboring astrocytes. If we consider the aforementioned results regarding opposite glucose uptake changes after glutamate exposure, then the most probable sequence of events occurring on activation involving glutamatergic synapses is an oxidative use of pre-existing extracellular lactate by neurons that are then supplied upon prolonged stimulation by lactate produced by astrocytes. Measurements performed in vivo have already provided observations consistent with this view.

Magnetic resonance spectroscopy in humans has revealed an initial "dip" in lactate concentration on activation in the corresponding brain region that could correspond to its oxidative use by neurons (60). Moreover, rapid and synchronized decreases in extracellular lactate 
levels monitored with lactate-sensitive microelectrodes have been observed after electrical stimulation of the hippocampus in the rat (61). Although early or initial measurements revealed a transient decrease in lactate levels, observations made later usually found an increase in lactate or lactate "peaks" (62-64). This biphasic response is entirely consistent with a rapid oxidative burst in neurons sustained by extracellular lactate and later supplied via lactate production by astrocytes that replenish the extracellular pool.

\section{Cellular Distribution of LDH Isoforms and Monocarboxylate Transporters Supports the Concept of an Astrocyte-Neuron Lactate Shuttle}

It has been proposed that neurons behave as lactate "sinks" whereas astrocytes would act as lactate "source" within the brain parenchyma. The necessity of a direct transfer of lactate from astrocytes to neurons is alleviated by the existence of an extracellular lactate pool, as the extracellular lactate concentration was found to be approx $1 \mathrm{mM}$ in both rats and humans (65). After activation in a specific brain region, a net lactate transfer occurs as a result of early oxidative consumption in neurons and late production by astrocytes. This overall process forms what has been named the astrocyte-neuron lactate shuttle hypothesis (for review, see ref. 66). However, for this mechanism to operate, some key elements in the metabolism and transport of lactate are either required or must at least be indicative that the mechanism can occur. This is the case for the distribution of LDH isoforms.

$\mathrm{LDH}$ is a tetrameric enzyme formed from two types of subunits in the CNS: the muscle type (or M), and the heart type (or $\mathrm{H}$ ). Isoforms enriched in $\mathrm{H}$ subunits (e.g., LDH1 formed of four $\mathrm{H}$ subunits) are found highly expressed in tissues or cell types that consume lactate (e.g., heart), whereas isoforms with high $\mathrm{M}$ subunit content (e.g., LDH5 with four M subunits) are abundant in lactate-producing tissues or cells (e.g., fast-twitch muscle). It was discovered that neurons in the brain predominantly express the LDH1 isoform, whereas the LDH5 isoform is more abundant in astrocytes $(67,68)$.

Lactate is an hydrophilic substance and can not easily cross plasma membranes at physiological $\mathrm{pH}$; instead, it requires specific transporters. A family of monocarboxylate transporters was recently described (for review, see ref. 69). Among the 14 members identified on the basis of sequence homologies, only the first 4 monocarboxylate transporters (MCT1-MCT4) were capable of transporting monocarboxylates that include lactate, pyruvate, and ketone bodies. MCT1, -2, and -4 have been observed in the CNS (70).

MCT1 and MCT4 are abundantly expressed by astrocytes in various brain regions (70-76). In contrast, MCT2 is the major neuronal MCT (73-78). Considering that MCT2 exhibits a much higher affinity than MCT1 and, especially, MCT4, the reported distribution is consistent with export of lactate by astrocytes and neuronal lactate uptake. At the subcellular level, MCT2 was observed not only on dendrites and axons of neurons in various areas (78) but also localized in the postsynaptic density area of glutamatergic synapses but not GABAergic synapses $(77,79)$. Additionally, MCT2 was observed to colocalize with the GluR2/3 subunits of AMPA receptors in the postsynaptic density area and also within the postsynaptic spine head, forming an intracellular pool (79).

It was suggested that MCT2, together with AMPA receptors, could undergo a process of exo-/endocytosis at the plasma membrane of the postsynaptic density area, providing a mean to adjust energy substrate delivery with the level of postsynaptic activity (79). As a proof of principle, MCT2 expression in cultured neurons was enhanced through a viral vector-based approach (80). After exposure to glutamate, it was demonstrated that lactate oxidation was enhanced in neurons overexpressing MCT2, compared to untransfected 
cultures. Additionally, the possibility that the expression levels of MCTs could be controlled was recently demonstrated. It was shown that noradrenaline causes a transient increase in the expression of MCT2 in cultured neurons, an effect occurring at the translational, rather than at the transcriptional, level (81). This observation suggests that in addition to a rapid control of MCT2 expression at the plasma membrane, there may also be some long-term mechanisms to adapt lactate delivery to active neurons as a function of the global level of activity in a specific brain area.

\section{Astrocytes As the Origin of Specific Brain Imaging Signals and As a Putative Therapeutic Target in Neurodegenerative Diseases}

The demonstration that astrocytes represent a major site of glucose uptake (becoming particularly prominent following activation) has important implications for the interpretation of signals arising from functional brain imaging techniques. The first direct consequence is that for imaging performed with fluorodeoxyglucose combined with positron emission tomography, the signal obtained largely originates from astrocytes. Although generally it reflects neuronal activity because the mechanism in astrocytes is related to synaptic activity, there could be some situations where this relationship is altered (82-86).

A second important point concerns the impact of inhibitory vs excitatory activity on both energetics and brain imaging signals, which is an issue under much debate $(87,88)$. In cultured astrocytes, research showed that GABA (the major inhibitory neurotransmitter in the CNS) does not cause any change in glucose utilization, as opposed to the prominent effect of glutamate (20). These results were interpreted as an indication that inhibitory neurotransmission might not induce a similar imaging signal based on energetics, a view consistent with some observations made in vivo (89).
A new dimension was recently added to the contribution of astrocytes in brain imaging signals. It was observed that the metabolic response of astrocytes to glutamate (i.e., enhanced glucose utilization) propagates from one cell to another through a regenerative mechanism of glutamate release and re-uptake (90). This observation has important implications for the interpretation of brain imaging signals that might spread over larger areas than where they originate.

In a certain number of neurodegenerative diseases, deficits in brain energy metabolism were evidenced and often revealed by functional brain imaging. This is the case of Alzheimer's disease, for which deficits even preceded the appearance of the first symptoms, suggesting that it could be a contributing factor to, rather than a consequence of, cell loss. In parallel, indications that lactate can be neuroprotective in different conditions have started to emerge.

Therefore, lactate was shown to at least partly reverse the deleterious effect of glucose or oxygen deprivation (91-95), ischemia (96), or excitotoxicity $(97,98)$. Such observations gave rise to the concept that boosting neuroenergetics by enhancing lactate production by astrocytes, enhancing lactate consumption by neurons, or both might be beneficial and provide neuroprotection (99). This principle was directly tested experimentally, and it was discovered that transfecting a glucose transporter in astrocytes (that enhanced lactate production) or a MCT in neurons (that favored lactate consumed) was neuroprotective against an excitotoxic insult (80). Moreover, a combination of the two treatments was even more effective than each approach separately.

Further support for the idea that bolstering energy metabolism in astrocytes might represent a valuable therapeutic strategy was recently provided. It was shown that the ampakine CX546, belonging to a well-known family of cognitive enhancers and neuroprotective agents (100), enhances the metabolic response of astrocytes to glutamate (101). This effect, which might contribute to the neuroprotective effects of this class 
of compounds, points at the possibility of specifically targeting astrocytes and their energy metabolism as a prime therapeutic target-particularly for neurodegenerative diseases such as Alzheimer's disease.

\section{Acknowledgments}

Author's work is supported by the Swiss Fonds National de la Recherche Scientifique grant N³100A0-100679.

\section{References}

1. Attwell D. and Laughlin S. B. (2001). An energy budget for signaling in the grey matter of the brain. J. Cereb. Blood Flow Metab. 21, 1133-1145.

2. Rothman D. L., Behar K. L., Hyder F., and Shulman R. G. (2003). In vivo NMR studies of the glutamate neurotransmitter flux and neuroenergetics: implications for brain function. Annu. Rev. Physiol. 65, 401-427.

3. Wong-Riley M. T. T. (1989). Cytochrome oxidase: an endogenous metabolic marker for neuronal activity. Trends Neurosci. 12, 94-101.

4. Nehlig A. and Pereira de Vasconcelos A. (1993). Glucose and ketone body utilization by the brain of neonatal rats. Prog. Neurobiol. 40, 163-221.

5. Fox P. T., Raichle M. E., Mintun M. A., and Dence C. (1988). Nonoxidative glucose consumption during focal physiologic neural activity. Science 241, 462-464.

6. Morgello S., Uson R. R., Schwartz E. J., and Haber, R. S. (1995). The human blood-brain barrier glucose transporter (GLUT1) is a glucose transporter of gray matter astrocytes. Glia 14, 43-54.

7. Yu S. and Ding, W. G. (1998). The $45 \mathrm{kDa}$ form of glucose transporter 1 (GLUT1) is localized in oligodendrocyte and astrocyte but not in microglia in the rat brain. Brain Res. 797, 65-72.

8. Golgi C. (1886). Sulla Fina Anatomia degli Organi Centrali del Sistema Nervosa. Hoepli, Milano, pp. 214; footnote on p. 154.

9. Porter J. T. and McCarthy K .D. (1997). Astrocytic neurotransmitter receptors in situ and in vivo. Prog. Neurobiol. 51,439-455.

10. Gadea A. and Lopez-Colome A. M. (2001). Glial transporters for glutamate, glycine and
GABA I. Glutamate transporters. J. Neurosci. Res. 63, 453-460.

11. Gadea A. and Lopez-Colome A. M. (2001). Glial transporters for glutamate, glycine, and GABA II. GABA transporters. J. Neurosci. Res. 63, 461-468.

12. Gadea A. and Lopez-Colome A. M. (2001). Glial transporters for glutamate, glycine, and GABA III. Glycine transporters. J. Neurosci. Res. 64, 218-222.

13. Schipke C. G. and Kettenmann H. (2004). Astrocyte responses to neuronal activity. Glia 47, 226-232.

14. Haydon P. G. (2001). GLIA: listening and talking to the synapse. Nat. Rev. Neurosci. 2, 185-193.

15. Pellerin L. and Magistretti P. J. (2004). Neuroenergetics: calling upon astrocytes to satisfy hungry neurons. Neuroscientist 10, 53-62.

16. Pellerin L. and Magistretti P. J. (1994). Glutamate uptake into astrocytes stimulates aerobic glycolysis: a mechanism coupling neuronal activity to glucose utilization. Proc. Natl. Acad. Sci. USA. 91, 10,625-10,629.

17. Chatton J. Y., Marquet P., and Magistretti P. J. (2000). A quantitative analysis of L-glutamateregulated $\mathrm{Na}+$ dynamics in mouse cortical astrocytes: implications for cellular bioenergetics. Eur. J. Neurosci. 12, 3843-3853.

18. Pellerin L. and Magistretti P. J. (1997). Glutamate uptake stimulates $\mathrm{Na}+, \mathrm{K}+-$ ATPase activity in astrocytes via activation of a distinct subunit highly sensitive to ouabain. J. Neurochem. 69, 2132-2137.

19. Cholet N., Pellerin L., Magistretti P. J., and Hamel E. (2002). Similar perisynaptic glial localization for the $\mathrm{Na}+\mathrm{K}+$-ATPase alpha 2 subunit and the glutamate transporters GLAST and GLT-1 in the rat somatosensory cortex. Cereb. Cortex. 12, 515-525.

20. Chatton J. Y., Pellerin L., and Magistretti P. J. (2003). GABA uptake into astrocytes is not associated with significant metabolic cost: implications for brain imaging of inhibitory transmission. Proc. Natl. Acad. Sci. USA 100, $12,456-12,461$.

21. Loaiza A., Porras O. H., and Barros L. F. (2003). Glutamate triggers rapid glucose transport stimulation in astrocytes as evidenced by real-time confocal microscopy. J. Neurosci. 23, 7337-7342.

22. Akaoka H., Szymocha R., Beurton-Marduel P., Bernard A., Belin M.F., and Giraudon P. (2001). Functional changes in astrocytes by human Tlymphotropic virus type-1 T-lymphocytes. Virus Res. 78, 57-66. 
23. Ramos M., del Arco A., Pardo B., et al. (2003). Developmental changes in the Ca2+-regulated mitochondrial aspartate-glutamate carrier aralar1 in brain and prominent expression in the spinal cord. Dev. Brain Res. 143, 33-46.

24. Hertz L., Swanson R. A., Newman G. C., Marrif H., Juurlink B. H., and Peng L. (1998). Can experimental conditions explain the discrepancy over glutamate stimulation of aerobic glycolysis? Dev. Neurosci. 20, 339-447.

25. Peng L., Swanson R. A., Hertz L. (2001). Effects of L-glutamate, D-aspartate, and monensin on glycolytic and oxidative glucose metabolism in mouse astrocyte cultures: further evidence that glutamate uptake is metabolically driven by oxidative metabolism. Neurochem. Int. 38, 437-443.

26. Brunet J. F., Grollimund L., Chatton J.Y., et al. (2004). Early acquisition of typical metabolic features upon differentiation of mouse neural stem cells into astrocytes. Glia 46, 8-17.

27. Kasischke K. A., Vishwasrao H. D., Fischer P. J., Zipfel W. R., and Webb W. W. (2004). Neural activity triggers neuronal oxidative metabolism followed by astrocytic glycolysis. Science 305, 99-103.

28. Cholet N., Pellerin L., Welker E., et al. (2001). Local injection of antisense oligonucleotides targeted to the glial glutamate transporter GLAST decreases the metabolic response to somatosensory activation J. Cereb. Blood Flow Metab. 21, 404-412.

29. Voutsinos-Porche B., Bonvento G., Tanaka K., et al. (2003). Glial glutamate transporters mediate a functional metabolic crosstalk between neurons and astrocytes in the mouse developing cortex. Neuron 37, 275-286.

30. Voutsinos-Porche B., Knott G., Tanaka K., Quairiaux C., Welker E., and Bonvento G. (2003). Glial glutamate transporters and maturation of the mouse somatosensory cortex. Cereb. Cortex. 13, 1110-1121.

31. Nehlig A., Wittendorp-Rechenmann E., and Dao Lam C. (2004). Selective uptake of $\left[{ }^{14} \mathrm{C}\right] 2-$ deoxyglucose by neurons and astrocytes: highresolution microautoradiographic imaging by cellular ${ }^{14}$ C-trajectography combined with immunohistochemistry. J. Cereb. Blood Flow Metab. 24, 1004-1014.

32. Porras O. H., Loaiza A., and Barros L. F. (2004). Glutamate mediates acute glucose transport inhibition in hippocampal neurons. J. Neurosci. 24, 9669-9673.
33. Pellerin L. and Magistretti P. J. (2003). How to balance the brain energy budget while spending glucose differently. J. Physiol. 546, 325.

34. Véga C., Martiel J. L., Drouhault D., Burckhart M. F., and Coles J. A. (2003). Uptake of locally applied deoxyglucose, glucose and lactate by axons and Schwann cells of rat vagus nerve. J. Physiol. 546, 551-564.

35. Véga C., Poitry-Yamate C. L., Jirounek P., Tsacopoulos M., and Coles J. A. (1998). Lactate is released and taken up by isolated rabbit vagus nerve during aerobic metabolism. J. Neurochem. 71, 330-337.

36. Schurr A., Miller J. J., Payne R. S., and Rigor B. M. (1999). An increase in lactate output by brain tissue serves to meet the energy needs of glutamate-activated neurons. J. Neurosci. 19, 34-39.

37. Serres S., Bouyer J. J., Bezancon E., Canioni P., and Merle M. (2003). Involvement of brain lactate in neuronal metabolism. NMR Biomed. 16, 430-439.

38. Serres S., Bezancon E., Franconi J. M., and Merle M. (2004). Ex vivo analysis of lactate and glucose metabolism in the rat brain under different states of depressed activity. J. Biol. Chem. 279, 47,881-47,889.

39. Pellerin L. (2003). Lactate as a pivotal element in neuron-glia metabolic cooperation. Neurochem. Int. 43, 331-338.

40. McIlwain H. (1953). Substances which support respiration and metabolic response to electrical impulses in human cerebral tissues. J. Neurol. Neurosurg. Psychiatry 16, 257-266.

41. Ide T., Steinke J., and Cahill G. F., Jr. (1969). Metabolic interactions of glucose, lactate, and $\beta$-hydroxybutyrate in rat brain slices. Am. J. Physiol. 217, 784-792.

42. Fernandez E. and Medina J. M. (1986). Lactate utilization by the neonatal rat brain in vitro. Competition with glucose and 3-hydroxybutyrate. Biochem. J. 234, 489-492.

43. Vicario C., Arizmendi C., Malloch G., Clark J. B., and Medina J. M. (1991). Lactate utilization by isolated cells from early neonatal rat brain. J. Neurochem. 57, 1700-1707.

44. Tabernero A., Vicario C., and Medina J. M. (1996). Lactate spares glucose as a metabolic fuel in neurons and astrocytes from primary culture. Neurosci. Res. 26, 369-376.

45. McKenna M. C., Hopkins I. B., and Carey A. (2001). $\alpha$-cyano-4-hydroxycinnamate decreases both glucose and lactate metabolism in neurons and astrocytes: implications for lactate as 
an energy substrate for neurons. J. Neurosci. Res. 66, 747-754.

46. Honegger P., Braissant O., Henry H., et al. (2002). Alteration of amino acid metabolism in neuronal aggregate cultures exposed to hypoglycaemic conditions. J. Neurochem. 81, 1141-1151.

47. Larrabee M. G. (1983). Lactate uptake and release in the presence of glucose by sympathetic ganglia of chicken embryos and by neuronal and nonneuronal cultures prepared from these ganglia. J. Neurochem. 40, 1237-1250.

48. Larrabee M. G. (1992). Extracellular intermediates of glucose metabolism : fluxes of endogenous lactate and alanine through extracellular pools in embryonic sympathetic ganglia. $J$. Neurochem. 59, 1041-1052.

49. Larrabee M. G. (1995). Lactate metabolism and its effect on glucose metabolism in an excised neural tissue. J. Neurochem. 64, 1734-1741.

50. Larrabee M. G. (1996). Partitioning of CO2 production between glucose and lactate in excised sympathetic ganglia, with implications for brain. J. Neurochem. 67, 1726-1734.

51. McKenna M. C., Tildon J. T., Stevenson J. H., Boatright R., and Huang S. (1993). Regulation of energy metabolism in synaptic terminals and cultured rat brain astrocytes: differences revealed using aminooxyacetate. Dev. Neurosci. $15,320-329$.

52. McKenna M. C., Tildon J. T., Stevenson J. H., and Hopkins I. B. (1994). Energy metabolism in cortical synaptic terminals from weanling and mature rat brain: evidence for multiple compartments of tricarboxylic acid cycle activity. Dev. Neurosci. 16, 291-300.

53. McKenna M. C., Tildon J. T., Stevenson J. H., Hopkins I. B., Huang X., and Couto R. (1998). Lactate transport by cortical synaptosomes from adult brain: characterization of kinetics and inhibitor specificity. Dev. Neurosci. 20, 300-309.

54. Itoh Y., Esaki T., Shimoji K., et al. (2003). Dichloroacetate effects on glucose and lactate oxidation by neurons and astroglia in vitro and on glucose utilization by brain in vivo. Proc. Natl. Acad. Sci. USA 100, 4879-4884.

55. Bouzier-Sore A. K., Voisin P., Canioni P., Magistretti P. J., and Pellerin L. (2003). Lactate is a preferential oxidative energy substrate over glucose for neurons in culture. J. Cereb. Blood Flow Metab. 23, 1298-1306.

56. Bouzier A. K., Thiaudiere E., Biran M., Rouland R., Canioni P., and Merle M. (2000). The metabolism of [3-(13)C]lactate in the rat brain is spe- cific of a pyruvate carboxylase-deprived compartment. J. Neurochem. 75, 480-486.

57. Hassel B. and Brathe A. (2000). Cerebral metabolism of lactate in vivo: evidence for neuronal pyruvate carboxylation. J. Cereb. Blood Flow Metab. 20, 327-336.

58. Qu H., Haberg A., Haraldseth O., Unsgard G., and Sonnewald U. (2000). (13)C MR spectroscopy study of lactate as substrate for rat brain. Dev. Neurosci. 22, 429-436.

59. Smith D., Pernet A., Hallett W. A., Bingham E., Marsden P. K., and Amiel S. A. (2003). Lactate: a preferred fuel for human brain metabolism in vivo. J. Cereb. Blood Flow Metab. 23, 658-664.

60. Mangia S., Garreffa G., Bianciardi M., Giove F., Di Salle F., and Maraviglia B. (2003). The aerobic brain: lactate decrease at the onset of neural activity. Neuroscience 118, 7-10.

61. Hu Y. and Wilson G. S. (1997). A temporary local energy pool coupled to neuronal activity: fluctuations of extracellular lactate levels in rat brain monitored with rapid-response enzymebased sensor. J. Neurochem. 69, 1484-1490.

62. Prichard J., Rothman D., Novotny E., et al. (1991). Lactate rise detected by 1H NMR in human visual cortex during physiologic stimulation. Proc. Natl. Acad. Sci. USA 88, 5829-5831.

63. Fellows L. K., Boutelle M. G., and Fillenz M. (1993). Physiological stimulation increases nonoxidative glucose metabolism in the brain of the freely moving rat. J. Neurochem. 60, 1258-1263.

64. Frahm J., Kruger G., Merboldt K. D., and Kleinschmidt A. (1996). Dynamic uncoupling and recoupling of perfusion and oxidative metabolism during focal brain activation in man. Magn. Reson. Med. 35, 143-148.

65. Abi-Saab W. M., Maggs D. G., Jones T., et al. (2002). Striking differences in glucose and lactate levels between brain extracellular fluid and plasma in conscious human subjects: effects of hyperglycemia and hypoglycemia. J. Cereb. Blood Flow Metab. 22, 271-279.

66. Pellerin L. and Magistretti P. J. (2003). Food for thought: challenging the dogmas. J. Cereb. Blood Flow Metab. 23, 1282-1286.

67. Bittar P. G., Charnay Y., Pellerin L., Bouras C., and Magistretti P. J. (1996). Selective distribution of lactate dehydrogenase isoenzymes in neurons and astrocytes of human brain. J. Cereb. Blood Flow Metab. 16, 1079-1089.

68. Laughton J. D., Charnay Y., Belloir B., Pellerin L., Magistretti P. J., and Bouras C. (2000). Differential messenger RNA distribution of lactate 
dehydrogenase LDH-1 and LDH-5 isoforms in the rat brain. Neuroscience 96, 619-625.

69. Pierre K. and Pellerin L. (2005). Monocarboxylite transporters in the central nervous system: distribution, regulation and function. $J$. Neurochem. 94, 1-14.

70. Pellerin L., Begersen L., Halestrap A. P., and Pierre K. (2005). Cellular and subcellular distribution of monocarboxylate transporters in cultured brain cells and in the adult brain. J. Neurosci. Res. 79, 55-64.

71. Bröer S., Rahman B., Pellegri G., et al. (1997). Comparison of lactate transport in astroglial cells and monocarboxylate transporter 1 (MCT1) expressing Xenopus laevis oocytes: expression of two different monocarboxylate transporters in astroglial cells and neurons. J. Biol. Chem. 272, 30,096-30,102.

72. Hanu R., McKenna M., O’Neill A., Resneck W. G., and Bloch R. J. (2000). Monocarboxylic acid transporters, MCT1 and MCT2, in cortical astrocytes in vitro and in vivo. Am. J. Physiol. 278, C921-C930.

73. Pierre K., Pellerin L., Debernardi R., Riederer B. M., and Magistretti P. J. (2000). Cell-specific localization of monocarboxylate transporters, MCT1 and MCT2, in the adult mouse brain revealed by double immunohistochemical labeling and confocal microscopy. Neuroscience 100, 617-627.

74. Bergersen L., Rafiki A., and Ottersen O. P. (2002). Immunogold cytochemistry identifies specialized membrane domains for monocarboxylate transport in the central nervous system. Neurochem. Res. 27, 89-96.

75. Debernardi R., Pierre K., Lengacher S., Magistretti P. J., and Pellerin L. (2003). Cell-specific expression pattern of monocarboxylate transporters in astrocytes and neurons observed in different mouse brain cortical cell cultures. J. Neurosci. Res. 73, 141-155.

76. Rafiki A., Boulland J. L., Halestrap A. P. Ottersen O. P., and Bergersen L. (2003). Highly differential expression of the monocarboxylate transporters MCT2 and MCT4 in the developing rat brain. Neuroscience 122, 677-688.

77. Bergersen L., Waerhaug O., Helm J., et al.. (2001). A novel postsynaptic density protein: the monocarboxylate transporter MCT2 is colocalized with $\delta$-glutamate receptors in postsynaptic densities of parallel fiber-Purkinje cell synapses. Exp. Brain Res. 136, 523-534.

78. Pierre K., Magistretti P. J., and Pellerin L. (2002). MCT2 is a major neuronal monocar- boxylate transporter in the adult mouse brain. J. Cereb. Blood Flow Metab. 22, 586-595.

79. Bergersen L., Magistretti P .J., and Pellerin L. (2004). Selective postsynaptic co-localisation of MCT2 with AMPA receptor GluR2/3 subunits at excitatory synapses exhibiting AMPA receptor trafficking. Cerebral Cortex (Epub).

80. Bliss T. M., Ip M., Cheng E., et al. (2004). Dualgene, dual-cell type therapy against an excitotoxic insult by bolstering neuroenergetics. J. Neurosci. 24, 6202-6208.

81. Pierre K., Debernardi R., Magistretti P. J., and Pellerin L. (2003). Noradrenaline enhances monocarboxylate transporter 2 expression in cultured mouse cortical neurons via a translational regulation. J. Neurochem. 86, 1468-1476.

82. Mazziotta J. C., Phelps M. E., Pahl J. J., et al. (1987). Reduced cerebral glucose metabolism in asymptomatic subjects at risk for Huntington's disease. N. Engl. J. Med. 316, 357-362.

83. Reiman E. M., Caselli R. J., Yun L. S., et al. (1996). Preclinical evidence of Alzheimer's disease in persons homozygous for the epsilon 4 allele for apolipoprotein E. N. Engl. J. Med. 334, 752-758

84. Back T., Zhao W., and Ginsberg M. D. (1995). Three-dimensional image analysis of brain glucose metabolism-blood flow uncoupling and its electrophysiological correlates in the acute ischemic penumbra following middle cerebral artery occlusion. J. Cereb. Blood Flow Metab. 15, 566-577.

85. Bruehl C., Hagemann G., and Witte O. W. (1998). Uncoupling of blood flow and metabolism in focal epilepsy. Epilepsia. 39, 1235-1242.

86. Molnar M. J., Valikovics A., Molnar S., et al. (2000). Cerebral blood flow and glucose metabolism in mitochondrial disorders. Neurology 55, 544-588.

87. Tagamets M. A. and Horwitz B. (2001). Interpreting PET and fMRI measures of functional neural activity: the effects of synaptic inhibition on cortical activation in human imaging studies. Brain Res. Bull. 54, 267-273.

88. Arthurs O. J. and Boniface S. (2002). How well do we understand the neural origins of the fMRI BOLD signal? Trends Neurosci. 25, 27-31.

89. Waldvogel D., van Gelderen P., Muellbacher W., Ziemann U., Immisch I., and Hallett M. (2000). The relative metabolic demand of inhibition and excitation. Nature 406, 995-998.

90. Bernardinelli Y., Magistretti P. J., and Chatton J. Y. (2004). Astrocytes generate Na+-mediated metabolic waves. Proc. Natl. Acad. Sci. USA 101, 14,937-14,942. 
91. Izumi Y., Benz A. M., Katsuki H., and Zorumski C. F. (1997). Endogenous monocarboxylates sustain hippocampal synaptic function and morphological integrity during energy deprivation. J. Neurosci. 17, 9448-9457.

92. Schurr A., Payne R. S., Miller J. J., and Rigor B. M. (1997). Brain lactate, not glucose, fuels the recovery of synaptic function from hypoxia upon reoxygenation: an in vitro study. Brain Res. 744, 105-111.

93. Schurr A., Payne R. S., Miller J. J., and Rigor B. M. (1997). Glia are the main source of lactate utilized by neurons for recovery of function posthypoxia. Brain Res. 774, 221-224.

94. Cater H. L., Benham C. D., and Sundstrom L. E. (2001). Neuroprotective role of monocarboxylate transport during glucose deprivation in slices culture of rat hippocampus. J. Physiol. (Lond) 531, 459-466.

95. Cater H. L., Chandratheva A., Benham C. D., Morrison B., and Sundstrom L. E. (2003). Lactate and glucose as energy substrates during, and after, oxygen deprivation in rat hippocampal acute and cultured slices. J. Neurochem. 87, 1381-1390.

96. Schurr A., Payne R. S., Miller J. J., Tseng M. T., Rigor B. M. (2001). Blockade of lactate trans- port exacerbates delayed neuronal damage in a rat model of cerebral ischemia. Brain Res. 895, 268-272.

97. Mendelowitsch A., Ritz M. F., Ros J., Langemann H., and Gratzl O. (2001). 17ß-Estradiol reduces cortical lesion size in the glutamate excitotoxicity model by enhancing extracellular lactate: a new neuroprotective pathway. Brain Res. 901, 230-236.

98. Ros J., Pecinska N., Alessandri B., Landolt H., Fillenz M. (2001). Lactate reduces glutamateinduced neurotoxicity in rat cortex. J. Neurosci. Res. 66, 790-794.

99. Sapolsky R. M. (2003). Neuroprotective gene therapy against acute neurological insults. Nat. Rev. Neurosci. 4, 61-69.

100. Lynch G. (2004). AMPA receptor modulators as cognitive enhancers. Curr. Opin. Pharmacol. 4, 4-11.

101. Pellerin L. and Magistretti P. J. (2005). Ampakine CX546 bolsters energetic response of astrocytes: a novel target for cognitive-enhancing drugs acting as AMPA receptor modulators. J. Neurochem., 92, 668-677. 